\title{
Depth Profiling: Fundamental Aspects and Recent Progress
}

\author{
Siegfried HOFMANN \\ Max-Planck-Institut für Metalforschung, Institut für Werkstoffwissenschaft, \\ Seestr. 92, D-7000 Stuttgart 1, FRG
}

(Received July 12, 1990, Accepted August 4, 1990)

\begin{abstract}
Depth profiling by sputteirng in combination with surface analysis has become a most effective way to determine the depth distribution of composition in thin films. The principles of the method and its fundamental capabilities and limitations are briefly discussed. Various phenomena, the most important of which are due to ion beam induced changes of surface roughness and composition, limit the experimentally achievable depth resolution. Recent progress in studying the influence of sample characteristics and experimental parameters like ion beam energy and incidence angle as well as sample rotation on depth resolution and its dependence on the sputtered depth serves as a guideline for optimized profiling conditions. With these, even for metallic samples and sputtered depths approaching micrometer dimension, a depth resolution of a few nanometers can be obtained. Knowledge of the depth resolution function allows deconvolution of a measured, normalized profile in order to obtain the true depth distribution of composition.
\end{abstract}

\section{Introduction}

Modern materials science and technology is based on knowledge and control of phenomena and processes in very small dimensions. This fact requires an effective and reliable micro-local analysis. In particular, the distribution of chemical composition with respect to its distance from a surface or interface is of decisive importance for the properties and behaviour of thin film structures as well as for fundamental research on reactions at surfaces and interfaces. Of the numerous techniques which are capable of providing such an information, the combination of microsectioning by ion sputtering with surface analysis techniques based on ion spectroscopies (SIMS, SNMS, ISS) or electron spectroscopies (AES, XPS) is most frequently applied. The main reason is the small depth of information of these techniques of the order of $1 \mathrm{~nm}$ or below, which is a prerequisite for obtaining high in-depth resolution. On the other hand, sputtering operates on an atomic scale and in principle offers a resolved depth in this range. Bombardment of a solid surface with primary particles, in general noble gas ions of $0.5-5 \mathrm{keV}$ energy, causes removal of matter from the surface. By continuous sputtering, layers beneath the topmost layer are subsequently exposed. Therefor ethere are two ways to obtain the in-depth distribution of composition as a function of the sputtered depth: either by analysis of the sputtered matter, like in SIMS and SNMS, or by analyzing the remaining surface, like in AES, XPS and ISS. Both methods should give the same results if all components are removed with the same sputtering yield. For preferential sputtering of a component they give complementary results. However, the sputter erosion process is independent of the analysis method and can therefore be considered under a common point of view.

The aim of the paper is to give a brief summary of the fundamental aspects, the present state and recent progress in understanding and optimization of depth profiling by sputtering. For the physical principles of the analysis methods and for further details the reader is referred to respective review articles. ${ }^{1-6)}$

\section{Principles of Depth Profiling by Sputtering}

The aim of quantitative depth profiling is the determination of the local composition $X_{\mathrm{i}}$ of the components $i$ as a function of its depth $\mathbf{z}$ beneath the original surface, $X_{\mathrm{i}}=f(z)$. The experimental in- 
formation, however, consists in an element- or compound-specific signal intensity $I_{\mathrm{i}}$ as a function of the sputtering time $t, I_{\mathrm{i}}=f(t)$. Therefore, the basic problem to solve is a correct conversion of the intensity-sputtering time relation to the desired composition-depth relation. This requires both a knowledge of the quantification parameters and of the instantaneous sputtering rate. Assuming that such a calibration has been achieved, the resulting profile will still deviate from the original in-depth distribution due to ion beam induced alteration of surface composition and topography. This deviation is usually characterized by the depth resolution $\Delta z$ (i.e. resolved depth) which is a figure of merit of the accuracy of a depth profile. Therefore optimization of $\Delta z$ is indispensable for the achievement of high quality depth profiling, and is the subject of numerous papers. ${ }^{1-6)}$

\subsection{Calibration of the Depth Scale}

For a constant sputtering rate $\dot{z}=d z / d t$, the sputtered depth $z$ is proportional to the sputtering time, i.e. $z(t)=\dot{z} \cdot t$. Since $z$ is proportional to the sputter yield $Y_{\mathrm{M}}$ and to the primary ion current density $j_{\mathrm{p}}$, a measure of the latter by a Faraday cup and taking $Y_{\mathrm{M}}$ from literature data gives the sputtering rate. However, this is only a rough estimate since $Y_{M}$ depends on various parameters, e.g. on energy and incidence angle of the ions and on the surface composition. ${ }^{7-10)}$ For practical purposes, a metal or an oxide layer (most often $\mathrm{Ta}_{2} \mathrm{O}_{5} / \mathrm{Ta}$ ) of known thickness is sputter profiled under identical conditions and the obtained sputtering rate has to be corrected with respect to the material under study. Another method is the measurement of the depth of the crater, formed after a sufficiently extended sputtering time, by a mechanical stylus or by optical interferometry. ${ }^{11,12)}$ In both cases, only an average value of $\dot{z}$ is obtained. Because $\dot{z}$ may vary with $z$ due to the dependence of the sputtering yield on the instantaneous surface composition, a nonlinear time-depth relationship may occur. In this case, the changing sputtering rate can only be determined in situ during the sputtering experiment, e.g. interferometrically ${ }^{13}$ or by determination of the mass of the sputtered matter. ${ }^{14)}$ For thin metallic films, the X-ray emission is a measure of the residual film thickness and can be used for this purpose. ${ }^{15)}$ The principle advantage of the methods detecting the sputtered species, e.g. SIMS and SNMS, is that they analyze a constant fraction of the total matter sputtered, provided that the elemental analysis is quantitative for all constituents. ${ }^{10,16)}$ Often a linear relation between the sputtering rate and the instantaneous composition is approximately fulfilled when sputtering through an A/B interface. This was shown in AES depth profiling of $\mathrm{Ni} / \mathrm{Cr}$ multilayers with $\mathrm{N}_{2}^{+}$ ions. ${ }^{4,17)}$ In general, however, auxiliary methods as described above are necessary to establish the true depth scale in sputter profiling with electron spectroscopies.

\subsection{Calibration of the Composition}

The general problem of quantification of surface analysis methods is the subject of special publications (see e.g. ${ }^{1,10,18,19)}$ ). Whereas sputter erosion and quantitative analysis are directly coupled in SIMS and SNMS, both processes are separated in AES and XPS, with ISS being a somewhat intermediate case. Thus, high resolution scanning Auger microscopy (SAM) can be used to analyze the edge of the sputtered crater for depth profiles with high resolution. ${ }^{3)}$ The main advantage of SIMS $^{10)}$ and SNMS ${ }^{2)}$ are their large dynamic range which allows trace analysis and their capability of hydrogen detection. On the other hand SIMS is difficult to quantify because the ionization probability strongly varies with composition. Whereas sputtering with reactive ions (e.g. $\mathrm{O}_{2}^{+}$) is a means to "normalize" the surface chemistry, ${ }^{10)}$ post-ionization of sputtered neutrals (i.e. SNMS) has been shown to allow a practically matrix-independent calibration. ${ }^{2,16)}$ AES and XPS are only slightly influenced by original and sputtering induced surface roughness and by composition changes. ${ }^{3,18)}$

The depth of information is of great importnace in depth profiling. ${ }^{3,4)}$ In this respect, ISS is outstanding because of its confinements to the detection of the first monolayer. ${ }^{2,19)}$ In SIMS, the origin of the sputtered particles may extend to some monolayers, ${ }^{10)}$ and in AES and XPS the attenuation length of Auger- or photoelectrons is generally in the range of 0.3 to $3 \mathrm{~nm} .^{20,21)}$ In AES, the electron backscattering factor must additionally be taken into account. ${ }^{18,22)}$

It can easily be shown that the attenuation length (or escape depth) of the electrons $\lambda$ leads to an exponential decrease of the A signal at an A/B interface and to a respective increase of the $B$ signal for ideal microsectioning. ${ }^{3,4)}$ The influence of the backscattering factor is superimposed and most pronounced when A and B differ widely in their atomic numbers. ${ }^{22)}$ If $\mathrm{B}$ is of higher atomic number than A, the signal of A increases in front of the interface and a steepening of the measured profile 
results, which was demonstrated e.g. for the oxygen AES profile at a $\mathrm{Ta}_{2} \mathrm{O}_{5} / \mathrm{Ta}$ interface. ${ }^{4,23)}$ In general, the electron escape depth has a larger influence on profile broadening than the backscattering factor.

Quantitative analysis often depends on the chemical bonding state of the elements which is directly observed in XPS. ${ }^{24)}$ This can be of particular importance in AES, where peak shape alterations due to chemical bonding may change the peak-to peak-height values of the spectra in the derivative mode. ${ }^{25)}$ A simple quantitative separation is only possible for special cases (e.g. $\mathrm{Si} / \mathrm{SiO}_{2}$, $\mathrm{Al} / \mathrm{Al}_{2} \mathrm{O}_{3}$ with about 12 and $15 \mathrm{eV}$ difference between the metallic and oxide peak). In general, only a peak shape change is observed and more elaborate methods like least squares fitting of standard spectra or factor analysis have to be used to extract the chemical information in a quantitative manner. ${ }^{26,27)}$ This was recently shown for thin oxide layers on $\mathrm{FeCrNi}$ alloys, ${ }^{28,29)}$ where the ion beam induced decomposition of the oxide is negligible. If this is not the case (e.g. for $\mathrm{Ta}_{2} \mathrm{O}_{5}$ ), the altered layer with an oxide and a metallic component is obtained in AES depth profiling. ${ }^{3)}$

\subsection{Depth Resoluton in Sputter Profiling}

Sputter profiling inevitably leads to a certain broadening of the measured profile with respect to the true, original one. ${ }^{30)}$ Mathematically, it can be described by a resolution function $g\left(z-z^{\prime}\right)$, by which each composition $X(z)$ is affected. If the integral over $g\left(z-z^{\prime}\right)$ is normalized to one, the

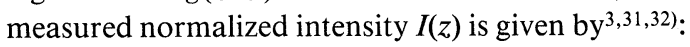

$$
I(z)=\int_{-\infty}^{+\infty} X\left(z^{\prime}\right) \cdot g\left(z-z^{\prime}\right) d z^{\prime}
$$

Knowledge of the resolution function allows a deconvolution of eq. (1) to retrieve the compositional in-depth distribution $X(z) .^{3,32)}$ Due to the many parameters which contribute to the resolution function (see below), it is difficult to predict. The most simple and general assumption, which is most often a good approximation of experimental data, is a Gaussian function for $g\left(z-z^{\prime}\right)$. This means that a sharp step function concentration profile is broadened in a Gaussian integral or error function, which is defined by its standard deviation $\sigma$. The generally accepted definition for the depth resolution is $\Delta z=2 \sigma$ which corresponds to the difference of the $z$ values between $84 \%$ and $16 \%$ of the maximum intensity. $3,4,30,32,33)$ Fig. 1 visualizes schematically profile broadening and the definition

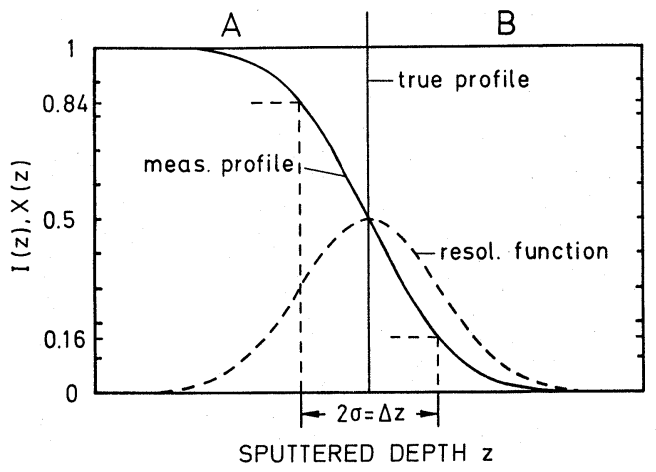

Fig. 1 Profile broadening and definition of the depth resolution $/ \Delta z$ for a Gaussian resolution function. The $\Delta z(84-16 \%)$ definition is approximately valid also in the case of asymmetric profile broadening, i.e. non-Gaussian resolution functions.

of the depth resolution.

According to the above definition, the depth resoution $\Delta z$ can be measured directly by sputter profiling through a sharp interface $A / B$ and is given by the apparent interface width. This is usually done with anodized oxide layers ${ }^{23)}$ or with metallic evaporation or sputter deposited layers. ${ }^{34)}$ Single sandwich layers of infinitesimal thickness will directly reproduce the resolution function (see Fig. 1), whereas those with a finite thickness $d$ can be described by a superposition of error functions. ${ }^{3,34,35)}$ for small $d$, the measured normalized peak intensity of a single sandwich layer, $I_{\mathrm{s}} / I_{0}$, drops below 1 and can be used to obtain the depth resolution according to ${ }^{34.35)}$ :

$$
\frac{I_{s}}{I_{0}}=\operatorname{erf}\left[\frac{d}{2^{1 / 2} \cdot \Delta z}\right]
$$

Frequently multilayer sandwich structures are used which allow the determination of the depth dependence of $\Delta z$ in one single run..$^{4,6,16,36-44)}$ In this case, it has been shown that for a single layer thickness $d$, for $\Delta z / d<0.4$ the single interface measurement of $\Delta z$ can be applied (see Fig. 1), whereas for $0.4<\Delta z / d<2$ the amplitude $I_{\mathrm{m}}$ between the maxima and minima is less than $100 \%$ $\left(=I_{0}\right)$ and $I_{\mathrm{m}} / I_{0}$ is approximately given by ${ }^{3,35)}$ :

$$
\frac{I_{\mathrm{m}}}{I_{0}} \simeq 1-2 \cdot\left[\operatorname{erf}\left[\frac{3 d}{2^{1 / 2} \cdot \Delta z}\right]-\operatorname{erf}\left[\frac{d}{2^{1 / 2} \cdot \Delta z}\right]\right]
$$

Equation (2b) has been found particularly useful for the extraction of $\Delta z$ values in $\mathrm{Ni} / \mathrm{Cr}$ multilayer 


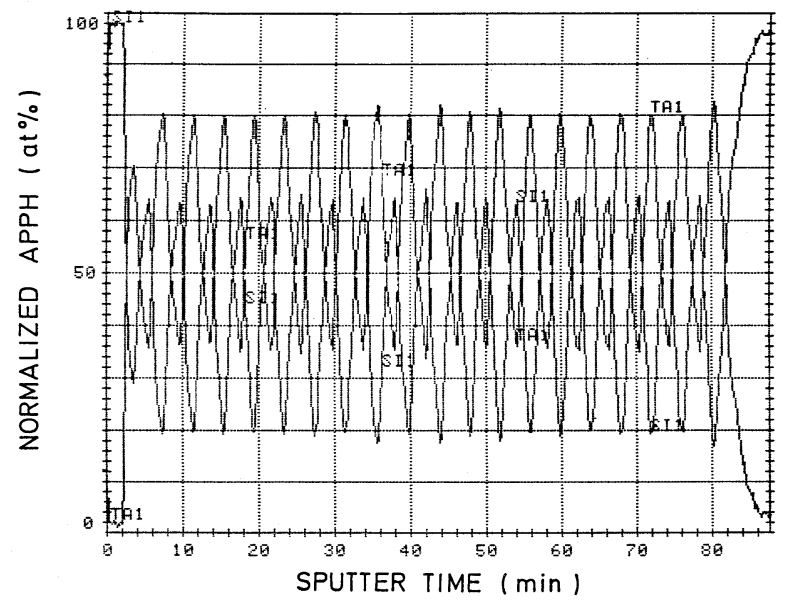

a)

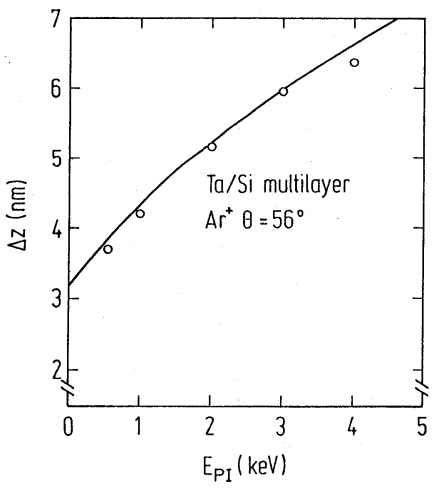

b)

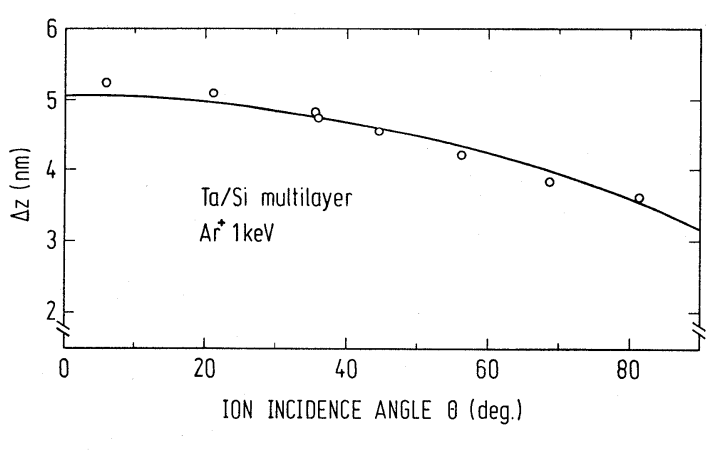

c)

Fig. 2 AES depth profiling of a Ta/Si multilayer structure composed of 20 alternating layers with a double layer $(\mathrm{Ta}+\mathrm{Si})$ thickness of $10 \mathrm{~nm}$ with $\mathrm{Ar}^{+}$ions (rastered beam, $\left.3 \times 3 \mathrm{~mm}^{2}\right) .^{45}$ )

a) typical depth profile for $1 \mathrm{keV} \mathrm{Ar}{ }^{+}$ions at an incidence angle of $\Theta=68^{\circ}$. b) depth resolution $\Delta z$ as a function of the ion energy for $\Theta=56^{\circ}$. c) depth resolution $\Delta z$ as a function of the ion incidence angle $\Theta$ for $1 \mathrm{keV} \mathrm{Ar}^{+}$ions.

samples as a function of primary ion energy, incidence angle and of surface roughness. ${ }^{37-39)}$ It can be extended to include sandwich layers of different thickness. ${ }^{36)}$ A recent example of multilayer sputtering is shown in Fig. 2. ${ }^{45)}$ The samples consist of a sequence of 20 alternating layers of $\mathrm{Ta}$ and $\mathrm{Si}$ with a period of $10 \mathrm{~nm}\left(d_{\mathrm{Ta}} \approx 3 \mathrm{~nm}, d_{\mathrm{Si}} \approx 7 \mathrm{~nm}\right)$ and were sputtered with $\mathrm{Ar}^{+}$ions at diffeent energies and incidence angles.

\section{Distortional Effects in Depth Profiling}

There are a large number of effects which will distort a depth profile. In general, they are recognized by a degradation of the depth resolution and may be roughly divided in instrumental factors, effects depending on sample composition and mor- phology and the fundamental effects of ion beamsample interactions. A compilation of the most important contributions to $\Delta z$ is given in Table $1 .^{3,4)}$

Instrumental factors depend on the proper setup of the instrument. Low residual gas pressure $(<$ $10^{-8} \mathrm{~Pa}$ ) and high purity sputtering gas are a prerequisite to avoid contamination effects. ${ }^{46)}$ Most important is the attainment of a flat bottom of the sputtering crater and an analysis in this region. Generally, a restered ion beam gives optimum results. In SIMS, raster gating is necessary to avoid crater edge effects. ${ }^{47)}$ Conventional XPS is less suited for high resolution profiling, because of the large analyzed area in $\mathrm{mm}$ dimensions. ${ }^{47)}$ Small area XPS $(\leq 150 \mu \mathrm{m})$ is preferable. The situation is favorable in SAM with a small focussed electron 
Table 1 Survey of the main distortional effects in sputter profiling

\begin{tabular}{l|l}
\hline \hline $\begin{array}{l}\text { Instrumental } \\
\text { Factors }\end{array}$ & $\begin{array}{l}\text { adsorption from residual gas atmosphere } \\
\text { redeposition of sputtered species } \\
\text { impurities in ion beam } \\
\text { non-uniform ion beam intensity } \\
\text { information depth of analysis method }\end{array}$ \\
\hline $\begin{array}{l}\text { Sample } \\
\text { Characteristics }\end{array}$ & $\begin{array}{l}\text { Original surface roughness } \\
\text { crystalline structure and defects } \\
\text { alloys, compounds, } \\
\text { second phases } \\
\text { insulators }\end{array}$ \\
\hline $\begin{array}{l}\text { Ion Beam } \\
\text { Effects }\end{array}$ & $\begin{array}{l}\text { primary ion implantation } \\
\text { atomic mixing } \\
\text { sputtering induced roughness } \\
\text { preferential sputtering and } \\
\text { decomposition of compounds } \\
\text { enhanced diffusion and segregation } \\
\text { charging of insulators }\end{array}$ \\
\hline
\end{tabular}

beam of a few $\mu$ m diameter $\left(d_{\mathrm{e}}\right)$ or less. In this case, the depth resolution is approximately given by:

$$
\Delta z_{\mathrm{I}} \approx d_{\mathrm{e}} \cdot \frac{\partial\left(\bar{j}_{\mathrm{p}} / \overline{j_{\mathrm{p}}}\right)}{\partial x} \cdot z
$$

where $j_{\mathrm{p}}$ is the primary ion current as a function of the lateral coordinate $x, \overline{j_{\mathrm{p}}}$ is the mean primary ion current and $d_{\mathrm{e}}$ is the electron beam diameter. On the other hand, the crater edge of a static ion beam of normally Gaussian-like lateral distribution of $j_{\mathrm{p}}$ can be used to obtain a depth profile by lateral electron beam scanning (crater edge profiling ${ }^{3,48,49)}$ ).

It is seen from eq. (3) that $\Delta z_{\mathrm{I}}$ increases proportional to the sputtered depth $z$. No observable $z$ dependence means exclusion of an instrumental effect (see e.g. Fig. 2a). A useful test of a well adjusted instrumental setup is profiling through a $\mathrm{Ta}_{2} \mathrm{O}_{5} / \mathrm{Ta}$ sample of known thickness, where $\Delta z$ should be less than $2 \mathrm{~nm} .{ }^{23)}$ Even for small inhomogeneities of the ion beam intensity, $\Delta z_{\mathrm{I}}$ can be prevalent at higher depth due to its depth proportionality. This is evident from results of Magee and Honig as shown in Fig. 3. ${ }^{50}$

The depth of information depends on the analysis method and on the sample composition. It is optimum in low energy ISS, where only the first monolayer is detected, and in SIMS or SNMS with low ion energy. For higher energies $(>500 \mathrm{eV})$, the mean escape depth for sputtered particles increases as shown by Monte Carlo simulations of Ishitani and Shimizu. ${ }^{51)}$ In electron spectroscopies, the electron escape depth $\lambda$ can be taken into account, e.g. by taking $\lambda$ values from Seah and Dench. ${ }^{20)}$ Model

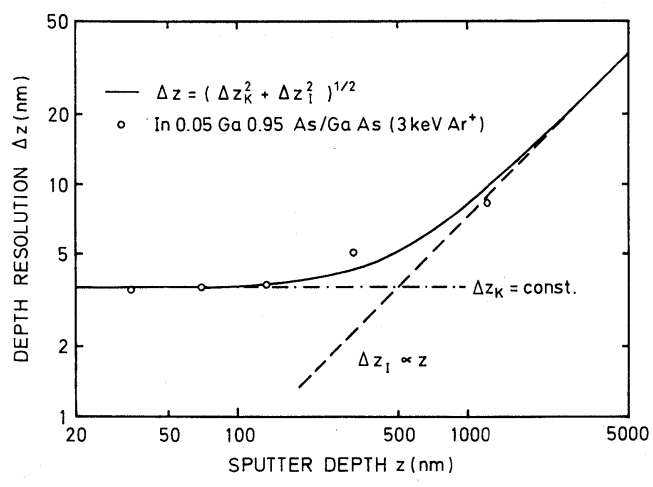

Fig. 3 Typical dependence of the total depth resoution $\Delta z$ on depth $z$ as predicted by eq. (5), fitted to measured data of Magee and Honig ${ }^{50}$ obtained by SIMS profiling of $\operatorname{In}_{0.05} \mathrm{Ga}_{0.95} \mathrm{As} / \mathrm{GaAs}$ interfaces with $3 \mathrm{keV} \mathrm{Ar}^{+}$ions.

calculations of the $\lambda$-effect ${ }^{3,4,52,53)}$ were experimentally tested with considerable success. ${ }^{15,23,54,55)}$ Since the measured intensity is proportional to $X(z) \cdot \exp (-z / \lambda)$, the resolution function $g\left(z-z^{\prime}\right)$ in eq. (1) can be replaced by this exponential function. With the intensity normalized to $I_{0}=I$ $(X=1)$ the solution of the integral gives ${ }^{3,4,52)}$ :

$$
X(z)=\frac{I}{I_{0}}-\lambda \cdot \frac{d\left[\frac{I}{I_{0}}\right]}{d z}
$$

An example of such a correction of the $\lambda$-effect is shown in Fig. 4, where the measured sputtering profile of the Si plasmon peak at $2 \mathrm{keV}$ primary electron energy is used to profile the interface of a silicon layer on sapphire. ${ }^{54)}$ The value of $\lambda$ is 2.0 $\mathrm{nm}$, which can be obtained self-consistently if the distance from the interface is larger than the mixing zone $e^{15,54}$ (see below). Application of eq. (4) to the measured profile gives the steeper, $\lambda$-corrected profile which still contains other contributions to profile broadening. The average contribution of $\lambda$ to the total depth resolution has been shown to be $\Delta z_{\lambda} \approx 1.6 \lambda^{3,4,32,56)}$ while the $50 \%$ value of the measured profile is shifted by about $0.7 \lambda$ to lower depth (see Figs. 4, 5). It should be noted that the probability of the depth of origin of sputtered particles can also be approximated by an exponentially decaying function with depth and can be similarly taken into account, particularly for higher ion energies. ${ }^{51)}$

Sample characteristics are of considerable influence in depth profiling. The original surface 


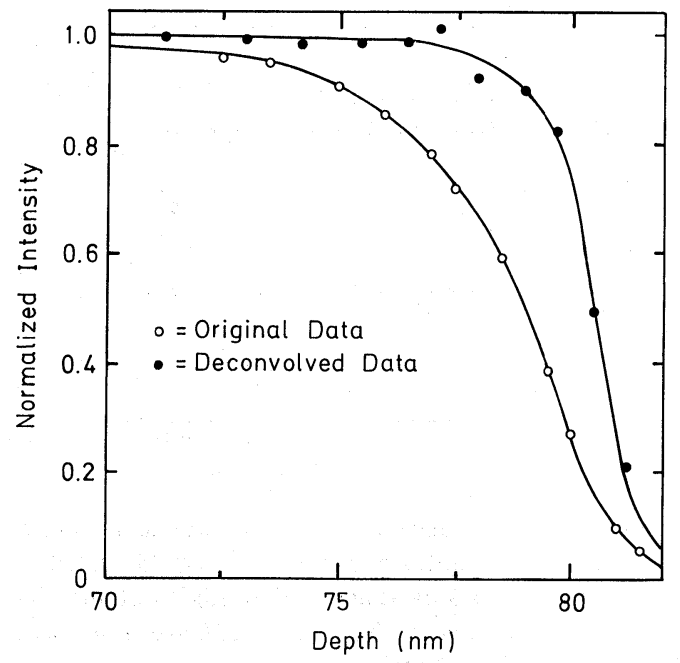

Fig. 4 Correction of the electron escape depth effect (O) according to eq. (4) with $\lambda=2 \mathrm{~nm}$ in the measured profile of the plasmon intensity $\left(E_{\text {kin }}=2\right.$ $\mathrm{keV})$ at a silicon-on-sapphire interface. From ref. ${ }^{54)}$

roughness causes an additional profile broadening which depends mainly on the ion beam angle of incidence and on the sputtered depth. ${ }^{38,39,57)}$ The decisive parameter is the distribution of the inclination angles of the microplanes on a surface, which is responsible for redeposition of the sputtered matter and shadowing of the primary ion beam. ${ }^{40,58)}$ Preferential sputtering of sample constituents is particularly detrimental in the case of second phases and leads to changes of surface composition which distort the measured profile. Most fundamental is the influence of the crystalline structure because of the dependence of the sputtering yield on the lattice orientation of metallic materials. ${ }^{59)}$ This is the main reason why semiconductors and oxides, which tend to amorphize under ion bombardment, show a better depth resolution as compared to metals. ${ }^{50}$.

Radiation induced effects result from the alterations of sample composition and morphology caused by ion bombardment. Besides primary ion implantation, the most fundamental effects are surface microroughening due to the statistical nature of the sputter erosion process ${ }^{30,61-63)}$ and atomic mixing within the collisional cascade. ${ }^{64-68)}$ The first effect results in a profile broadening which initially increases with the square root of depth $\left(\Delta z_{S} \propto z^{1 / 2}\right)$. It has been shown that both surface atom mobility ${ }^{62)}$ and site dependent emission

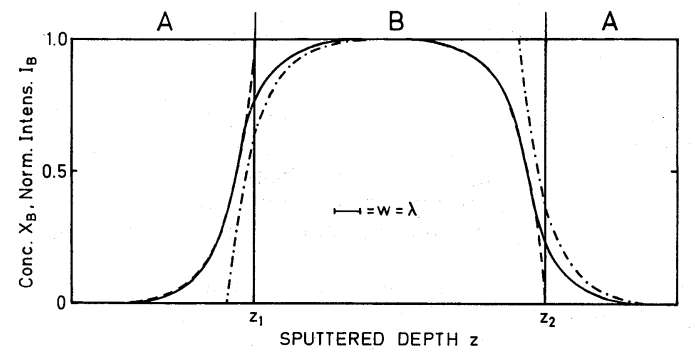

Fig. 5 Schematic plot of the influence of the electron escape depth $\lambda(---)$ and the atomic mixing zone $w(-\cdot \cdot)$ and their superposition on the measured profile (-) in AES/XPS depth profiling of $B$ at sharp $A / B$ and $B / A$ interfaces.

probability ${ }^{63)}$ counteract this effect and lead to a constant $\Delta z_{\mathrm{S}}$ of a few atomic layers depending on surface transport and binding energy parameters of the respective material. $32,62,63)$

Inherent to the sputtering process is the generation of a collisional cascade, resulting in an effective profile broadening $\left(\Delta z_{\mathrm{k}}\right)$ due to atomic mixing. ${ }^{64)}$ Initially, $\Delta z_{\mathrm{k}}$ is expected to vary with the square root of depth ${ }^{66,68)}$ until it saturates at a value which is of the order of the projected ion range, depending on projectile and materials parameters. ${ }^{64,69)}$ It can be treated by a diffusion model which reveals that it increases approximately with the square root of the ion energy as shown by Andersen. ${ }^{64)}$ The simplified assumption of a constant extension of the mixing zone, $w$, allows an analytical solution of the expected asymmetric shape of the measured profile at an interface. ${ }^{67)}$ It is proportional to $1-\exp \left[-\left(z-z_{1} / w+1\right]\right.$ and to $\exp \left[-\left(z-z_{2}\right) / w+1\right]$ at the front and rear side, respectively, of a concentration profile as depicted in Fig. 5. In AES profiling superposition of both the contribution of $\lambda$ and $w$ result in a good approximation of a more symmetric erf-like shape of the measured profile because of their inverse influence with sputtered depth. Evidence of the dependence of the zone of mixing on both ion energy and incidence angle is seen in Fig. 2 , where $\Delta z_{\mathrm{k}}$ is supposed to be the main contribution to $\Delta z$.

Together with atomic mixing, preferential sputtering ${ }^{70)}$ can occur and causes a further profile distortion. It leads to the formation of a layer with altered surface composition which has first been considered by Shimizu et al. ${ }^{71)}$ and gives for the stationary state of the surface composition $X_{\mathrm{A}}^{\mathrm{S}}, X_{\mathrm{B}}^{\mathrm{S}}$ of a binary alloy $(\mathrm{A}, \mathrm{B})$ : 


$$
\frac{X_{\mathrm{A}}^{\mathrm{s}}}{X_{\mathrm{B}}^{\mathrm{s}}}=\frac{Y_{\mathrm{B}}}{Y_{\mathrm{A}}} \cdot \frac{X_{\mathrm{A}}^{\mathrm{b}}}{X_{\mathrm{B}}^{\mathrm{b}}}
$$

where $X_{\mathrm{A}}^{\mathrm{b}}, X_{\mathrm{B}}^{\mathrm{b}}$ are the bulk molar fractions of $\mathrm{A}$ and $\mathrm{B}$ with the respective sputtering yields $Y_{\mathrm{A}}, Y_{\mathrm{B}}$. It should be noted, that in this case the sputtered matter analyzing methods (SIMS, SNMS) still represent the bulk composition, whereas the residual surface analyzing methods (AES, XPS, ISS) yield the surface composition. In the transient regime, both methods are prone to an apparent concentration change, even if there is none in the sample. Likewise, preferential sputtering causes distortions of the profile at an interface. Most obvious is the change in the sputtering time/depth relation. Therefore, an apparent asymmetry, i.e. a broadening of the $I(t)$ profile is observed at an A/B interface when $Y_{\mathrm{B}}<Y_{\mathrm{A}}$ and vice versa. ${ }^{4,17)}$

In many compounds, preferential sputtering of one component leads to decomposition, e.g. the reduction of oxides which causes profile distortions. ${ }^{24,72)}$ A number of additional effects may occur if one of the components has a strong tendency to surface segregation. In conjunction with increased mobility due to radiation induced defects this leads to further distortions of a profile which are difficult to predict. ${ }^{70,73-76)}$ In particular, charging of insulators may cause electrotransport which can even result in a complete surface depletion of a mobile positive ion, like $\mathrm{Na}^{+}$in glasses. ${ }^{77)}$ Electron stimulated desorption (ESD) can be encountered in AES profiling which acts like an additional selective sputtering, ${ }^{78)}$ while beam heating leads to enhanced interdiffusion. ${ }^{79)}$

Ion bombardment induced surface roughening at larger sputtered depth is often observed beyond the contributions of sputtering statistics and atomic mixing. Particularly in metallic materials, often cone-like features develop and lead to a degradation of depth resolution with the sputtered depth. ${ }^{57,80,81)}$ The angular dependence of the sputtering yield seems to play a decisive role, but also radiation induced diffusion and redeposition processes, as recently shown by Morishita et al. ${ }^{81)}$ In general, induced roughening is reduced with increasing ion beam angle and with higher ion mass and lower ion energies (see sect. 4).

The dependence of the sputtering yield on the lattice orientation is another cause of the depth dependence of $\Delta z$ in polycrystalline metallic materials. For a statistical orientation of small grains, it can be shown that $\Delta z \propto z^{1 / 2}$ at larger depth is expected, ${ }^{82}$ which is frequently observed. ${ }^{4,83}$ Semiconductors and oxides do not show such an effect because of their tendency to amorphize under ion bombardment. ${ }^{60)}$

In any sputter profiling experiment, all the above mentioned effects contribute to profile broadening to an extent which depends on a large variety of parameters. It has been shown ${ }^{4,83)}$ that if these contributions $\Delta z_{\mathrm{j}}$ are independent, their superposition is governed by subsequent convolutions of the original profile, i.e. they add up in quadrature to the total, experimentally obtainable $\Delta z$ given by ${ }^{3,4)}$ :

$$
\Delta z=\left[\sum_{j}\left(\Delta z_{\mathrm{j}}\right)^{2}\right]^{1 / 2}
$$

Equation (6) can be used to extract the extent of different contributions to the measured $\Delta z$. For example, the data in Fig. 2b, c can be rationalized by superposition of a constant contribution $\Delta z_{0}$ of about $3 \mathrm{~nm}$ and an incidence angle and primary energy dependent contribution, supposed to be mainly due to atomic mixing, of about $3.9 \mathrm{~nm}$ at 1 $\mathrm{keV} \mathrm{Ar}^{+}$and normal incidence. ${ }^{83)}$ Likewise, the data in Fig. $3^{50)}$ can be interpreted by a superposition of a constant atomic mixing term $\left(\Delta z_{\mathrm{k}}=3.6\right.$ $\mathrm{nm}$ at $3 \mathrm{keV} \mathrm{Ar}^{+}$) and an instrumental contribution $\Delta z_{\mathrm{l}}$. The latter varies linearly with depth according to eq. (3), giving $\Delta z_{\mathrm{I}}=0.007 \cdot z$, i.e. about $0.7 \%$ mean deviation from a perfectly flat crater bottom $^{3,84)}$

As seen from eq. (6), it is most important to reduce the preponderant contribution to $\Delta z$ to obtain a marked improvement of the quality of depth profiles.

\section{Optimized Profiling Conditions}

Optimized sputtering conditions in practice always are a compromise between the given sample characteristics and the instrumental possibilities. A survey of the most fundamental parameters is given in Table 2 in the light of the conclusions from section 3. Optimum cleanliness of the analysis system with respect to residual gas atmosphere and bombarding ion beam as well as its constant intensity in the analyzed area are obvious necessities. Low ion energy is required for minimizing the ion range (i.e. atomic mixing), as is a high ion incidence angle. In practice both measures may lead to an intolerable reduction of the sputtering rate. Morerover, a high incidence angle $\left(>60^{\circ}\right)$ is detrimental in the case of rough surfaces. An example of the influence of the incidence angle $\Theta$ on $\Delta z$ for smooth and rough $\mathrm{Ni} / \mathrm{Cr}$ multilayers and for 
Table 2 Survey of Optimized Sputter Depth Profiling Conditions

\begin{tabular}{|c|c|}
\hline $\begin{array}{l}\text { Sample } \\
\text { Ambient }\end{array}$ & $\begin{array}{l}\text { low residual reactive gas pressure } \\
\left(<10^{-8} \mathrm{~Pa}\right) \\
\text { "free" sample mount }\end{array}$ \\
\hline Ion Beam & $\begin{array}{l}\text { constant uniform intensity (scanning) } \\
\text { low beam energy ( } \leq 1 \mathrm{keV}) \\
\text { high mass ion species (or reactive species) } \\
\text { large incidence angle for smooth sample } \\
\left(>60^{\circ}\right) \\
\text { low incidence angle for rough sample } \\
\left(<60^{\circ}\right) \\
\text { two differently inclined beams and /or } \\
\text { sample rotation }\end{array}$ \\
\hline $\begin{array}{l}\text { Analyzing } \\
\text { Conditions }\end{array}$ & $\begin{array}{l}\text { analyzed area centered in and } \\
\text { small against sputtered area } \\
\text { minimized information depth }\end{array}$ \\
\hline $\begin{array}{l}\text { Sample } \\
\text { Characteristics }\end{array}$ & $\begin{array}{l}\text { smooth, polished surface } \\
\text { non-crystalline, no } 2 \text { nd phases } \\
\text { oxides, semiconductors } \\
\text { components with similar sputtering yields, } \\
\text { negligible diffusion and segregation and } \\
\text { good electrical and thermal conductivities }\end{array}$ \\
\hline
\end{tabular}

$\mathrm{Ta}_{2} \mathrm{O}_{5} / \mathrm{Ta}$ is shown in Fig. 6. ${ }^{85)}$ The maximum of $\Delta z$ for $\mathrm{NiCr}$ around $\Theta \approx 30^{\circ}$ is probably due to the preferred $\langle 111\rangle$ orientation of the sputter deposited layers which means a channeling direction for the primary ions at $\Theta=0$ and hence low sputtering yield and low change between different crystallites. ${ }^{59)}$ With increasing $\Theta$, an increasing number of crystallites is randomly oriented and the local change of the sputtering rate $z$ increases. This leads to increased $\Delta z$ as shown by computer simulations. ${ }^{36)}$ For higher angles of incidence, the probability of preferred orientations is markedly reduced as is the local change of $\dot{z}$. This is supposed to be the main reason for a decrease of $\Delta z$ with higher $\Theta$. As an example, FIg. 7 shows how $\Delta z$ and its dependence on $z$ is improved with low ion energy and high $\Theta$ in the case of smooth samples. ${ }^{85)}$ For rough samples, this effect is counterbalanced by redeposition and shadowing which increase with $\Theta$ and are prevalent for $\Theta>60^{\circ}$ (Fig. 6).

On the other hand, for $\mathrm{Ta}_{2} \mathrm{O}_{5} / \mathrm{Ta}$ no crystallinity effect is expected which results in a lower and depth-independent $\Delta z$ for $\Theta<60^{\circ}$ but still shows the typical roughness effect for large incidence angles. ${ }^{23)}$ Sputtering with reactive ions (e.g. $\mathrm{N}_{2}^{+}$) also helps to overcome the crystallinity effect thus lowering $\Delta z .^{17,80)}$ It has been shown that sputtering with two ion guns from different directions gives improved $\Delta z$ for $\mathrm{NiCr}$ multilayers due to further reduction of the local sputtering rate changes. ${ }^{86)}$ As

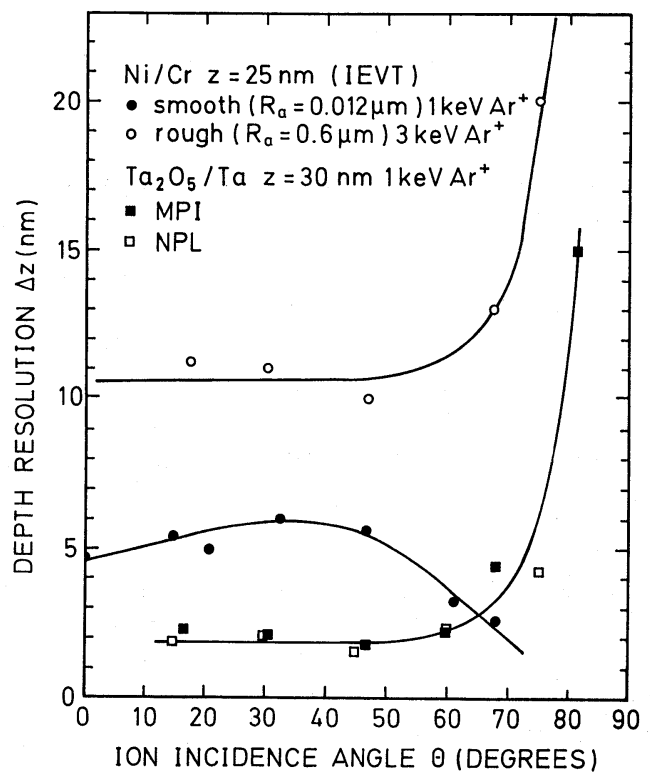

Fig. 6 Dependence of $\Delta z$ on the $\mathrm{Ar}^{+}$ion incidence angle $\Theta$ for $\mathrm{Ni} / \mathrm{Cr}$ multilayers with smooth $(\bullet)$ (roughness $\left.R_{\mathrm{a}} \leq 0.012 \mu \mathrm{m}\right)$ and rough $(O)\left(R_{\mathrm{a}}\right.$ $=0.6 \mu \mathrm{m})$ surfaces at $z=25 \mathrm{~nm}$ sputtered depth and for $\mathrm{Ta}_{2} \mathrm{O}_{5} / \mathrm{Ta}$ layers $(z=30 \mathrm{~nm})^{85)}\left(\right.$ IEVT $^{38)}$, $\mathrm{MPI}^{85)}$, NPL ${ }^{23)}$ ).

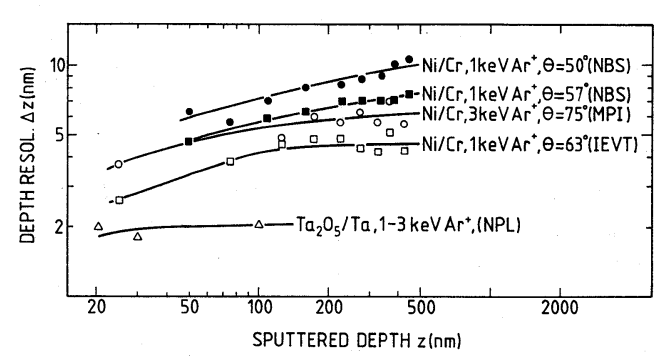

Fig. 7 Dependence of $\Delta z$ on the sputtered depth $z$ for Ni/Cr multilayers with smooth surfaces obtained at high ion incidence angle $\Theta$ by different laboratories $\left(\mathrm{NBS}^{42)}\right.$, MPI ${ }^{85)}, \mathrm{IEVT}^{85)}$ ) and compared with $\mathrm{Ta}_{2} \mathrm{O}_{5} / \mathrm{Ta}$ layers $\left(\mathrm{NPL}^{23)}\right)$ of different thicknesses. From ref. ${ }^{85)}$.

demonstrated by Zalar, ${ }^{87)}$ a similar and even more effective measure is rotation of the sample during ion bombardment. ${ }^{49,69,87)}$ Recently, Barna et al. reported that high angle sputtering with high energy $\mathrm{Ar}^{+}$ions $\left(10 \mathrm{keV}, \Theta=85^{\circ}\right)$ can be successfully applied to prevent sputtering induced roughness and consequently improve depth resolution. ${ }^{88)}$ Higher mass primary ions (e.g. $\mathrm{Xe}^{+}$) are generally preferable due to reduced extent of 
the mixing zone. ${ }^{15,64)}$

A prerequisite for optimized analysis conditions is the matching of the analyzed area with the center of the larger sputtered area to ensure a flat crater bottom within the former. A small information depth measns the use of low kinetic electron energy signals in AES and XPS and low primary ion energy in SIMS. Particularly in HF plasma excited SNMS, the primary ion energy is of the order of $10^{2} \mathrm{eV}$ which enhances the attainment of optimum $\Delta z .^{2,6,16)}$

The sample characteristics given in Table 2 describe a sample for which optimum profiling results can be expected with respect to the distortional effects mentioned in sect. 3. The above discussion gives some general guidelines for an appropriate choice of experimental parameters for optimum depth profiling and emphasize their dependence on the type of sample under study.

\section{Deconvolution of Sputtering Profiles}

In general, the raw data obtained as a sputtering profile, $I(t)$, deviate from the true in-depth distribution of the composition $X(z)$. The similarity of both depends on the degree of attainment of the optimized profiling conditions compiled in Table 2 . Only in favourable cases, the shape of the measured sputtering profile is a sufficiently precise image of the true, original profile. In any case the first step is the calibration of the intensity scale in terms of mormalized intensities or concentrations and that of the sputtering time scale in terms of sputtered depth. In particular, the latter procedure can be rather difficult if there is a marked dependence of the sputtering rate on composition. ${ }^{17)}$ In a second step the influence of all the effects which may have contributed to profile distortions (see sect. 3) must be considered. They can be represented by the resolution function $g(z-$ $\left.z^{\prime}\right)^{32,85)}$ in eq. (1). In some cases it is known or predictable (e.g. an exponential function for the effect of electron escape depth in AES and XPS) whereas in others (e.g. atomic mixing and preferential sputtering) more detailed estimations are necessary. A most general first order approximation for the resolution function is a Gaussian with the measured or estimated depth resolution $\Delta z=2 \sigma$. Then, the convolution integral eq. (1) can be solved with respect to $X(z)$ by usual deconvolution principles. ${ }^{3,32)}$ In practice, the method of true profile assumption and calculation of the normalized measured profile has been found very useful, particularly if $g\left(z-z^{\prime}\right)$ is a function of $z$.

A special case is encountered in depth profiling of the first few monolayers of a sample, where the resolution function depends mainly on th fundamental effects of ion mixing and micro-roughening on an atomic scale (the so-called sequential layer sputtering (SLS) model ${ }^{30}$ ) ) which can be described by a Poisson distribution. ${ }^{3,30}$ ) With the depth scale $z$ in atomic monolayers, the normalized measured Auger intensity $I(z)$ is given by ${ }^{72,89)}$ :

$$
\begin{aligned}
I(z)= & I_{0} \sum_{m=0}^{M} \sum_{n=1}^{N} X_{\mathrm{n}+\mathrm{m}}^{0} \frac{z^{(n-1)}}{(n-1) !} . \\
& \exp (-z) \cdot \exp (-m / \lambda)
\end{aligned}
$$

with $I_{0}$ a normalization factor, $X_{\mathrm{n}+\mathrm{m}}^{0}$ the molar fraction of the detected element in the layer $n+\mathrm{m}$; $z^{(\mathrm{n}-1)} /(n-1) ! \cdot \exp (-z)$ is the Poisson distribution term which accounts for the surface fraction of layer $n$ at depth $z$, and the exponential term $\exp (-m / \lambda)$ with $\lambda$ the electron escape depth accounts for the intensity of the Auger- or photoelectrons in this layer and below. Equation (7) has been successfully applied in many in-depth distribution studies. ${ }^{73,89,90)}$ As an example, the oxygen profile on a passive layer on a stainless steel surface is shown in Fig. 8. ${ }^{89)}$ The limitations and modifications of eq. (7) with respect to site dependent sputtering probability and the dependence of $\dot{z}$ and $\lambda$ on the composition are extensively discussed in ref. ${ }^{89)}$ Profiles of the other elements ( $\mathrm{Ni}, \mathrm{Cr}, \mathrm{Fe}$ ) were evaluated in a similar manner and allow a self-consistent test of the results when added up to the total concentration. Correction for preferential sputtering can be introduced in eq. (7) and was applied to describe the experimental results for oxygen profiles on $\mathrm{Nb}_{2}$ $\mathrm{O}_{5}$ surfaces. ${ }^{72)}$

Instead of elemental concentrations, component fractions disclosed e.g. by least squares fitting of standard spectra can also be used to determine the layered structure of oxides on alloy surfaces, as recently demonstrated in low temperature oxidation studies of $\mathrm{NiCrFe}$ alloys ${ }^{28,29)}$ and for passive layers on $\mathrm{FeCr} 18 .{ }^{91)}$

In spite of the many approximations, eq. (7) provides a useful deconvolution scheme for thin layers ( $\leq 20$ monolayers). For larger depth, a specifically measured or predicted resolution function has to be used in eq. (1).

\section{Summary and Conclusions}

Compositional depth profiling of thin films by ion sputtering and surface analysis at first requires 


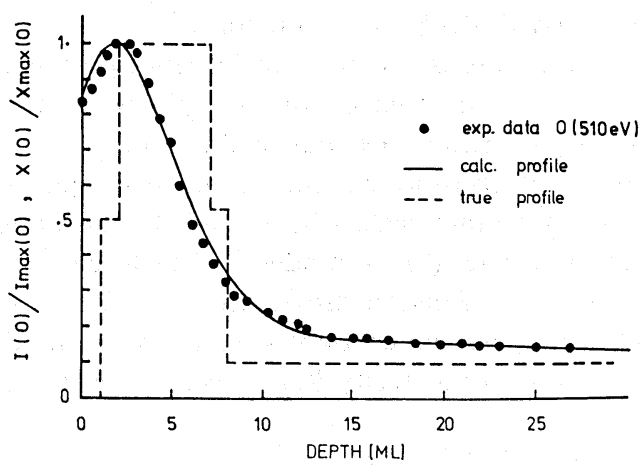

Fig. 8 Example of the evaluation of the true normalized in-depth distribution with eq. (7). Measured data $(\bullet)$ are from the $\mathrm{O}(510 \mathrm{eV})$ AES sputtering profile $\left(1 \mathrm{keV} \mathrm{Ar}^{+}\right)$of a passive layer on a FeCr18Ni8 stainless steel. The full drawn line is the optimum fit to the measured data as calculated from the normalized true in-depth distribution $\mathrm{X}(\mathrm{O}) / \mathrm{X}(\mathrm{O})_{\text {mas }}(---)(\lambda(\mathrm{O})=5.5$ monolayers $)$. The missing oxygen in the first layer is due to carbohydride contamination, and the non-vanishing oxygen content at larger depth can be explained by ion beam shadowing due to surface roughness. From ref. ${ }^{89)}$

quantification of the measured signal intensity and the sputtering time in terms of elemental concentrations and sputtered depth, respectively. Whereas the decisive parameters for quantitative AES and XPS are comparatively well known, SIMS often suffers from severe matrix effects. This situation is improved by the use of post-ionization techniques, as in SNMS. Nonlinearities in the time/depth scale conversion generally arise due to composition dependent sputtering rates. Preferential sputtering, radiation enhanced diffusion and segregation may cause variations in the measured concentration profile and are still difficult to predict. Metallic samples are particularly sensitive to sputtering induced surface roughnening which increases with the sputtered depth. The large number of parameters, depending on sample characteristics, instrumental factors and ion beam/sample interactions, lead to a broadening of the measured profile and can be represented by a respective resolution function, which is approximately characterized by the depth resolution obtained as the measured interface width on abrupt composition changes.

Recent advances in the study of the dependence of depth resolution on various experimental parameters like ion mass, energy and angle of incidence and sputtered depth have led to the conclu- sion that high mass, low energy and high angle of incidence ensure optimum depth resolution for samples with smooth surfaces. Dual beam sputtering and sample rotation during profiling yield further improvement. Even under optimized conditions, the most fundamental effects of ion beam/sample interaction, namely surface microroughening and atomic mixing still limit the obtainable depth resolution to a few nanometers. Careful experimental studies have shown, that the depth resolution can be pushed down to this limiting size.

Knowledge of the depth resolution or, more precisely, the depth resolution function allows the deconvolution of normalized measured profiles in order to obtain the true, original depth distribution of composition, with a precision in the atomic monolayer region for layers of some nanometer thickness.

\section{References}

1) H. w. Werner and R. P. H. Garten: Rep. Progr. Phys. 47 (1984) 221.

2) H. Oechsner, ed.: Thin Film and Depth Profile Analysis. Topics in Current Physis, Vol. 37. Springer, Berlin 1984.

3) S. Hofmann: Depth Profiling, chap. 2 in: D. Briggs and M. P. Seah, eds., Practical Surface Analysis with AES and XPS, Wiley, Chichester 1983, p. 148 (2nd ed. to be expected in 1990).

4) S. Hofmann: Surf. Interface Anal. 2 (1980) 148.

5) E. Zinner: J. Electrochem. Soc. 130 (1983) 199.

6) H. Oechsner: Vacuum 37 (1987) 763.

7) H. Oechsner: Appl. Phys. 8 (1975) 185.

8) M. P. Seah: Thin Solid Films 81 (1981) 279.

9) R. Behrisch, ed.: Sputtering by Ion Bombardment I, Topics in Appl. Phys. Vol. 47 and 52 (Springer Verlag, Heidelberg), Vol. I (1981) and Vol. II (1987).

10) A. Benninghoven, F. G. Rüdenauer and H. W. Werner: Secondary Ion Mass Spectrometry, Wiley, New York 1987.

11) J. M. Morabito and R. K. Lewis: Anal. Chem. 45 (1973) 869.

12) P. Laty, D. Seethanen and F. Degreve: Surf. Sci. 85 (1979) 533.

13) J. Kempf: Surf. Interface Anal. 4 (1982) 116.

14) J. H. Thomas and S. P. Sharma: J. Vac. Sci. Technol. 14 (1977) 1168.

15) J. Kirschner and H. W. Etzkorn: Appl. Surf. Sci. 3 (1979) 251.

16) A. Wucher and H. Oechsner: Fres. Z. Anal. Chem. 333 (1989) 470.

17) S. Hofmann and A. Zalar: Thin Solid Films 60 (1979) 201.

18) M. P. Seah: Vacuum 36 (1986) 399.

19) W. L. Baun: Surf. Interface Anal. 3 (1981) 243.

20) M. P. Seah and W. Dench: Surf. Interface Anal. 1 
(1979) 2 .

21) H. Tokutaka, K. Nishimori and H. Hayashi: Surf. Sci. 149 (1985) 349.

22) S. Ichimura and R. Shimizu: Surf. Sci. 112 (1981) 386.

23) C. P. Hunt and M. P. Seah: Surf. Interface Anal. 5 (1983) 199.

24) S. Hofmann and J. M. Sanz: J. Trace and Microprobe Techn. 1 (1982-83) 213.

25) H. H. Madden: J. Vac. Sci. Technol. 18 (1981) 677.

26) S. W. Gaarenstrom: Appl. Surf. Sci. 26 (1986) 561.

27) G. C. Nelson: J. Vac. Sci. Technol. A2 (1984) 1141.

28) J. Steffen and S. Hofmann: Surf. Interface Anal. 11 (1988) 617.

29) S. Hofmann and J. Steffen: Surf. Interface Anal. 14 (1989) 59.

30) S. Hofmann: Appl. Phys. 9 (1976) 59.

31) P. S. Ho and J. E. Lewis: Surf. Sci. 55 (1976) 335.

32) S. Hofmann and J. M. Sanz: in ref. 2) chap. 7, p. 141.

33) IUPAC Doc., G. H. Morrison, K. C. Cheng and M. Grasserbauer: Pure and Appl. Chemistry 51 (1979) 2243.

34) S. Hofmann: in: Proc. 7th Int. Vac. Congr. and 3rd Int. Conf. Sol. Surf., eds. A. Dobrozembsky et al., Berger, Vienna 1977, Vol. III, p. 2613.

35) S. Hofmann: Surf. Interface Anal. 8 (1986) 87.

36) S. Hofmann: in C. Powell, ed., Workshop on Quant. Surface Analysis, NBS Gaithersburg, Oct. 24, 1986.

37) S. Hofmann, J. Erlewein and A. Zalar: Thin Solid Films 43 (1977) 275.

38) A. Zalar and S. Hofmann: Nucl. Instr. a Meth. in Phys. Res. B18 (1987) 655.

39) A. Zalar and S. Hofmann: Vacuum 37 (1987) 169.

40) A. Zalar and S. Hofmann: J. Vac. Sci. Technol. A5 (1987) 1209.

41) R. J. Blattner, S. Nadel, C. A. Evans, A. J. Braundmeier and C. W. Magee: Surf. Interface Anal. 1 (1979) 32.

42) J. Fine, P. A. Lindfors, M. E. Gorman, R. L. Gerlach, B. Navinsek, D. F. Mitchell and G. P. Chambers: J. Vac. Sci. Technol. A3 (1985) 1413.

43) S. Sobue and F. Okuyama: J. Vac. Sci. Technol. A8 (1990) 785

44) K. Kajiwara and H. Kawai: Surf. Interface Anal. 15 (1990) 433

45) S. Hofmann, W. Hösler and R. von Criegern: Vacuum, to be publ.

46) H. J. Mathieu and D. Landolt: J. Microsc. Spectr. Electron 3 (1978) 113.

47) C. W. Magee, W. L. Harrington and R. E. Honig: Rev. Sci. Instr. 49 (1978) 477.

48) A. Zalar and S. Hofmann: Surf. Interface Anal. 2 (1980) 183.

49) S. Sobue, M. Tanemura and F. Okuyama: Surf. Interface Anal. 14 (1989) 451.

50) C. W. Magee and R. E. Honig: Surf. Interface Anal. 4 (1982) 35.

51) T. Ishitani and R. Shimizu: Appl. Phys. 6 (1975)
241.

52) H. Iwasaki and G. Nakamura: Surf. Sci. 57 (1976) 779.

53) F. Pons, J. LeHéricy and J. P. Langeron: Surf. Sci. 69 (1977) 565.

54) J. H. Thomas III and S. Hofmann: Surf. Interface Anal. 4 (1982) 156.

55) S. Mischler, H.-J. Mathieu and D. Landolt: Surf. Interface Anal. 11 (1988) 182.

56) S. Hofmann: Mikrochim. Acta Supp. 8 (1979) 71.

57) H. J. Mathieu, D. E. McClure and D. Landolt: Thin Solid Films 38 (1976) 281.

58) A. Zalar and S. Hofmann: Surf. Interface Anal. 12 (1988) 83.

59) H. E. Rosendaal: in ref. 9, Vol. I p. 219.

60) M. P. Seah and C. P. Hunt: Surf. Interface Anal. 5 (1983) 33.

61) R. Shimizu: Appl. Phys. 9 (1979) 425.

62) J. Erlewein and S. Hofmann: Thin Solid Films 69 (1980) L39.

63) M. P. Seah, J. M. Sanz and S. Hofmann: Thin Solid Films 81 (1981) 239.

64) H. H. Andersen: Appl. Phys. 18 (1979) 131.

65) S. Taikang, R. Shimizu, T. Okutani: Jap. J. Appl. Phys. 18 (1979) 1987.

66) U. Littmark and W. O. Hofer: Nucl. Instr. a. Meth. 168 (1980) 329.

67) Z. L. Liau, B. Y. Tsaur and J. W. Mayer: J. Vac. Sci. Technol. 16 (1979) 121.

68) K. Wittmaack: Vacuum 34 (1984) 119.

69) E-H. Cirlin, Y.-T. Cheng, P. Ireland and B. Clemens: Surf. Interface Anal. 15 (1990) 337.

70) R. Shimizu: Nucl. Instr. a. Meth. Phys. Res. B18 (1987) 486.

71) H. Shimizu, M. Ono and K. Nakayama: Surf. Sci. 36 (1973) 817.

72) J. M. Sanz and S. Hofmann: Surf. Interface Anal. 6 (1984) 78

73) D. G. Swartziager, S. B. Ziemecki and M. J. Kelley: J. Vac. Sci. Technol. 19 (1981) 185.

74) R. Kelly: Surf. Interface Anal. 7 (1985) 1.

75) T. Koshikawa and K. Goto: Nucl. Instr. a. Meth. Phys. Res. B18 (1987) 504.

76) N. Q. Lam: Surf. Interface Anal. 12 (1988) 65.

77) F. Ohuchi, M. Ogino, P. H. Holloway and C. G. Pantano: Surf. Interface Anal. 2 (1980) 85.

78) J. Ahn, C. R. Perleberg, D. L. Wilcox, J. W. Coburn and H. F. Winters: J. Appl. Phys. 46 (1975) 4581.

79) S. Hofmann and A. Zalar: Thin Solid Films 56 (1979) 337.

80) M. Tanemura and F. Okuyama: Thin Solid Films 165 (1988) 193.

81) S. Morishita, M. Tanemura, Y. Fujimoto and F. Okuyama: Appl. Phys. A46 (1988) 313.

82) D. Marton and J. Fine: Thin Solid Films 151 (1987) 433.

83) S. Hofmann: Appl. Phys. 13 (1977) 205.

84) H. W. Werner: Surf. Interface Anal. 4 (1982) 1.

85) S. Hofmann: Surf. Interface Anal. 9 (1986) 3. 
86) S. Hofmann and A. Zalar: Surf. Interface Anal. 10 (1987) 7 .

87) A. Zalar: Thin Solid Films 124 (1985) 223.

88) A. Barna, P. B. Barna and A. Zalar: Surf. Interface Anal. 12 (1988) 144.

89) J. M. Sanz and S. Hofmann: Surf. Interface Anal. 8
(1986) 147.

90) G. Lorang, F. Basile, M. Da Cunha Belo and J.-P. Langeron: Surf. Interface Anal. 12 (1988) 424.

91) J. Steffen and S. Hofmann: Frex. Z. Anal. Chem. 333 (1989) 408. 